\title{
NEW RESULTS ABOUT THE H-MEASURE OF A SET
}

\author{
Alina Bărbulescu \\ "Ovidius" University \\ Faculty of Mathematics and Informatics \\ 8700 Constanta \\ Romania \\ abarbulescu@univ-ovidius.ro
}

Abstract The aim of this paper is to generalize our results ([1], [2]) related to the Hasudorff measure of a plane set.

Keywords: Hausdorff measure, $\delta$ - class Lipschitz function, equivalence.

\section{Introduction}

We denote by $R^{n}$ the Euclidean $n$ - dimensional space and by $d(E)$ the diameter of a set $E \subset R^{n}$.

Definition 1.1 If $r_{0}>0$ is a fixed number, a continuous function $h(r)$, defined on $\left[0, r_{0}\right)$, nondecreasing and such that $\lim _{r \rightarrow 0} h(r)=0$ is called a measure function. If $\delta \in R_{+}, E \subset R^{n}$ is a bounded set, the Hausdorff $h$-measure of $E$ is defined by:

$$
H_{h}(E)=\lim _{\delta \rightarrow 0} \inf \sum_{i} h\left(\rho_{i}\right)
$$

inf being considered over all coverings of $\mathrm{E}$ with a countable number of spheres of radii $\rho_{i} \leq \delta$.

Definition $1.2 f: D\left(\subset R^{n}\right) \rightarrow \bar{R}$ is a $\delta$ - class Lipschitz function if

$$
|f(x+\alpha)-f(x)| \leq M|\alpha|^{\delta}, x \in D, \alpha \in R^{n}, x+\alpha \in D, M>0 .
$$

Definition 1.3 Let $\varphi_{1}, \varphi_{2}>0$ be functions defined in a neighborhood of $0 \in R^{n}$. We say that $\varphi_{1}$ and $\varphi_{2}$ are equivalent and we denote by:

The original version of this chapter was revised: The copyright line was incorrect. This has been corrected. The Erratum to this chapter is available at DOI: 10.1007/978-0-387-35690-7_44 
$\varphi_{1} \sim \varphi_{2}$, for $x \rightarrow 0$, if there exist $r>0, Q>0$, satisfying:

$$
\frac{1}{Q} \varphi_{1}(x) \leq \varphi_{2}(x) \leq Q \varphi_{1}(x),(\forall) x \in R^{n},|x|<r .
$$

An analogous definition can be given for $x \rightarrow \infty$. In this case, $\varphi_{1} \sim \varphi_{2}$ means that the previous inequalities have place in all the space.

Definition 1.4 The graph of the function $f:[0,1] \longrightarrow \bar{R}$ is the set:

$$
\Gamma=\{(x, f(x)) \mid x \in[0,1]\} .
$$

\section{Known results}

Theorem 2.1 If $h$ is a measure function such that

$$
h(t)^{\sim} t^{p}, p \geq 2,
$$

and $f:[0,1] \rightarrow \bar{R}$ is a $\delta$ - class Lipschitz function, with $\delta \in[0,1]$, then: $H_{h}(\Gamma)<+\infty$. In the same hypothesis about $h$ and $f$, the result remains true if $p \geq 1$ and $\delta>1$.

Consider

$$
g(x)=\left\{\begin{array}{cc}
2 x, & 0 \leq x<\frac{1}{2} \\
-2(x-1), & \frac{1}{2} \leq x<\frac{3}{2} \\
2(x-2), & \frac{3}{2} \leq x<2
\end{array}\right.
$$

and:

$$
f(x)=\sum_{i=1}^{\infty} \lambda_{i}^{-\delta} g\left(\lambda_{i} x\right),(\forall) x \in[0,1],
$$

where $\left\{\lambda_{i}\right\}_{i \in N^{*}}$ is a sequence of positive numbers.

\section{Theorem 2.2}

a. If $f$ is the function defined in (4), $\delta \in[0,1], \varepsilon>1,\left\{\lambda_{i}\right\}_{i \in N^{*}}$ is a sequence of positive numbers, such that $\lambda_{i+1}>\varepsilon \lambda_{i},(\forall) i \in N^{*}$ and $h$ is a measure function, such that: $h(t)^{\sim} t^{p}, p \geq 2$, then: $H_{h}(\Gamma)<+\infty$.

b. In the same hypothesis about $h, f$ and $\left\{\lambda_{i}\right\}_{i \in N^{*}}$, the result remains true if $\delta>1$ and $\varepsilon>1$. 


\section{New results}

Theorem 3.1 If $h$ is a measure function such that

$$
h(t)^{\sim} e^{t} t^{p}, p \geq 2,
$$

and $f:[0,1] \rightarrow \bar{R}$ is a $\delta$ - class Lipschitz function, with $\delta \in[0,1]$, then: $H_{h}(\Gamma)<+\infty$. The result remains true if $p \geq 1$ and $\delta>1$.

Proof. The first part of the proof follows that of [4].

First, we suppose that the coefficient in the Lipschitz inequality (1) can be taken 1 , so that to any $x$ corresponds an interval $(x-k, x+k)$ such that, for any $x+\alpha$ of this interval:

$$
|f(x+\alpha)-f(x)| \leq|\alpha|^{\delta} .
$$

Because $[0,1]$ is a compact set, there exists a finite set of overlapping intervals covering $(0,1)$ :

$$
\left(0, k_{0}\right),\left(x_{1}-k_{1}, x_{1}+k_{1}\right), \ldots,\left(x_{n-1}-k_{n-1}, x_{n-1}+k_{n-1}\right),\left(1-k_{n}, 1\right) .
$$

If $c_{i}$ are arbitrary points, satisfying:

$$
\begin{aligned}
& c_{1} \in\left(0, x_{1}\right), c_{i} \in\left(x_{i-1}, x_{i}\right), i=1,2, \ldots, n-1, c_{n} \in\left(x_{n-1}, 1\right) \\
& c_{i} \in\left(x_{i-1}-k_{i-1}, x_{i-1}+k_{i-1}\right) \frown\left(x_{i}-k_{i}, x_{i}+k_{i}\right), i=1, \ldots, n-1 .
\end{aligned}
$$

we have: $0<c_{1}<x_{1}<c_{2}<x_{2}<\ldots<x_{n-1}<c_{n}<1$.

The oscillation of $f(x)$ in the interval $\left(c_{i-1}, c_{i}\right)$ is less than $2\left(c_{i}-c_{i-1}\right)^{\delta}$ and thus the part of the curve corresponding to the interval $\left(c_{i-1}, c_{i}\right)$ can be enclosed in a rectangle of height $2\left(c_{i}-c_{i-1}\right)^{\delta}$ and of base $c_{i}-c_{i-1}$, and consequently in $\left[2\left(c_{i}-c_{i-1}\right)^{\delta-1}\right]+1$ squares of side $c_{i}-c_{i-1}$ or in the number of circles of radius $\frac{c_{i}-c_{i-1}}{\sqrt{2}}$ circumscribed about each of these squares.

We denoted by $[x]$ the integer part of $\mathrm{x}$.

Given an arbitrary $r \in\left(0, \frac{1}{2}\right)$ we can always assume: $c_{i}-c_{i-1}<r$, $i=2,3, \ldots, n$.

Denote by $C_{r}$ the set of all the above circles and consider

$$
\begin{aligned}
\sum_{C_{r}} h(2 r) & =\sum_{C_{r}}\left\{\frac{h(2 r)}{e^{2 r}(2 r)^{p}} \cdot e^{2 r}(2 r)^{p}\right\} . \\
r & \in\left(0, \frac{1}{2}\right), e^{2 r} \in(1, e)
\end{aligned}
$$


We have to estimate $\sum_{C_{r}}(2 r)^{p}$. The sum of the terms corresponding to the interval $\left(c_{i-1}, c_{i}\right)$ is:

$$
\begin{aligned}
& S=\left\{\left[2\left(c_{i}-c_{i-1}\right)^{\delta-1}\right]+1\right\}\left\{\left(c_{i}-c_{i-1}\right) \sqrt{2}\right\}^{p} \Leftrightarrow \\
& S=2^{\frac{p}{2}}\left(c_{i}-c_{i-1}\right)^{p}\left\{\left[2\left(c_{i}-c_{i-1}\right)^{\delta-1}\right]+1\right\} \text {. } \\
& S \leq 2^{\frac{p}{2}}\left(c_{i}-c_{i-1}\right)^{p}\left\{2\left(c_{i}-c_{i-1}\right)^{\delta-1}+1\right\} \Rightarrow \\
& S \leq 2^{\frac{p}{2}+1}\left(c_{i}-c_{i-1}\right)^{p+\delta-1}+2^{\frac{p}{2}}\left(c_{i}-c_{i-1}\right)^{p} \\
& c_{i}-c_{i-1}<1, p \geq 2, \delta \in[0,1] \Rightarrow \\
& \left\{\begin{array}{c}
\left(c_{i}-c_{i-1}\right)^{p}<c_{i}-c_{i-1} \\
p+\delta-1 \geq 1 \Rightarrow\left(c_{i}-c_{i-1}\right)^{p+\delta-1}<c_{i}-c_{i-1}
\end{array}\right.
\end{aligned}
$$

From (8) and (9) it results:

$$
\begin{gathered}
S \leq 2^{\frac{p}{2}+1}\left(c_{i}-c_{i-1}\right)+2^{\frac{p}{2}}\left(c_{i}-c_{i-1}\right)=3 \cdot 2^{\frac{p}{2}}\left(c_{i}-c_{i-1}\right) \Rightarrow \\
\sum_{C_{r}}(2 r)^{p} \leq \sum_{i=2}^{n} 3 \cdot 2^{\frac{p}{2}}\left(c_{i}-c_{i-1}\right)=3 \cdot 2^{\frac{p}{2}} \sum_{i=2}^{n}\left(c_{i}-c_{i-1}\right) \leq 3 \cdot 2^{\frac{p}{2}} \Leftrightarrow \\
\sum_{C_{r}}(2 r)^{p} \leq 3 \cdot 2^{\frac{p}{2}}
\end{gathered}
$$

Using the definition 2 and the relations (7) and (10), (6) gives:

$$
\sum_{C_{r}} h(2 r)=\sum_{C_{r}}\left\{\frac{h(2 r)}{(2 r)^{p}}(2 r)^{p}\right\}<Q e \sum_{C_{r}}(2 r)^{p} \leq 3 \cdot 2^{\frac{p}{2}} \cdot Q
$$

where $Q>0$ and $r \in\left(0, \frac{1}{2}\right)$, small enough.

Then $H_{h}(\Gamma)<+\infty$.

If $M \neq 1$, then $\sum_{C_{r}} h(2 r) \leq 3 \cdot 2^{\frac{p}{2}} \cdot Q M \Rightarrow H_{h}(\Gamma)<+\infty$.

If $p \geq 1$ and $\delta>1$, then:

$$
\begin{aligned}
& c_{i}-c_{i-1}<1, p \geq 1, \delta \geq 1 \Rightarrow \\
& \Rightarrow\left\{\begin{array}{l}
\left(c_{i}-c_{i-1}\right)^{p}<c_{i}-c_{i-1} \\
p+\delta-1 \geq 1 \Rightarrow\left(c_{i}-c_{i-1}\right)^{p+\delta-1}<c_{i}-c_{i-1}
\end{array}\right.
\end{aligned}
$$

and the proof is the same as above if we replace the relation (9) with (11). 
Theorem 3.2 If $h$ is a measure function such that

$$
h(t) \sim P(t) e^{T(t)},
$$

where $P$ and $T$ are polynomials with positive coefficients:

$$
\begin{aligned}
& P(t)=a_{1} t+a_{2} t^{2}+\ldots+a_{p} t^{p}, p \geq 1 \\
& T(t)=b_{0}+b_{1} t+\ldots+a_{m} t^{m},
\end{aligned}
$$

$f:[0,1] \rightarrow \bar{R}$ is a $\delta$ - class Lipschitz function, with $\delta \geq 1$, then: $H_{h}(\Gamma)<+\infty$. The result is also true if $p \geq 2, a_{1}=0$ and $\delta \in[0,1]$.

Proof. The first part follows that of the previous theorem. We have to estimate the sum $\sum_{C_{r}} h(2 r)$, for $r \in\left(0, \frac{1}{2}\right)$.

$$
\sum_{C_{r}} h(2 r)=\sum_{C_{r}} \frac{h(2 r)}{P(2 r) e^{T(2 r)}} \cdot P(2 r) e^{T(2 r)}<Q e^{\sum_{k=0}^{m} \frac{b_{k}}{2^{k}}} \sum_{C_{r}} P(2 r),
$$

because we have used the fact that $r \in\left(0, \frac{1}{2}\right)$ and (12).

Now, we estimate $\sum_{C_{r}} P(2 r)$. The sum of the terms corresponding to the interval $\left(c_{i-1}, c_{i}\right)$ is:

$$
S=\left\{\left[2\left(c_{i}-c_{i-1}\right)^{\delta-1}\right]+1\right\} \sum_{k=1}^{p} a_{k}\left(\left(c_{i}-c_{i-1}\right) \sqrt{2}\right)^{k},
$$

where $[x]$ is the integer part of $\mathrm{x}$.

$$
\begin{gathered}
S \leq\left\{2\left(c_{i}-c_{i-1}\right)^{\delta-1}+1\right\} \sum_{k=1}^{p} a_{k}\left(c_{i}-c_{i-1}\right)^{k} 2^{k / 2} \Leftrightarrow \\
S \leq 2^{\frac{p}{2}} \max _{k=1, p} a_{k}\left\{2\left(c_{i}-c_{i-1}\right)^{\delta-1}+1\right\} \sum_{k=1}^{p}\left(c_{i}-c_{i-1}\right)^{k} \Leftrightarrow \\
S \leq 2^{\frac{p}{2}} \max _{k=1, p} a_{k} \sum_{k=1}^{p}\left\{2\left(c_{i}-c_{i-1}\right)^{k+\delta-1}+\left(c_{i}-c_{i-1}\right)^{k}\right\} .
\end{gathered}
$$

If $p, \delta \geq 1$, then $k+\delta-1 \geq 1$ and $\left(c_{i}-c_{i-1}\right)^{k+\delta-1} \leq c_{i}-c_{i-1}$; thus:

$$
S \leq 3 \cdot 2^{\frac{p}{2}} \max _{k=1, p} a_{k} \sum_{k=1}^{p}\left(c_{i}-c_{i-1}\right)
$$

and it results, from (13):

$$
\sum_{C_{r}} h(2 r)<Q e^{\sum_{k=0}^{m}} \frac{b_{k}}{2^{k}} \cdot 3 \cdot 2^{\frac{p}{2}}
$$


where $Q>0$ and $r \in\left(0, \frac{1}{2}\right)$, small enough.

Then $H_{h}(\Gamma)<+\infty$.

If $p \geq 1, \delta \in[0,1)$, then

$$
1 \leq k-1 \leq k+\delta-1<k
$$

and

$$
\left(c_{i}-c_{i-1}\right)^{k}<\left(c_{i}-c_{i-1}\right)^{k+\delta-1} \leq c_{i}-c_{i-1}
$$

Thus

$$
S \leq 3 \cdot 2^{\frac{p}{2}} \max _{k=2, p} a_{k} \sum_{k=2}^{p}\left(c_{i}-c_{i-1}\right)
$$

and, analogous, it results that $H_{h}(\Gamma)<+\infty$.

\section{Theorem 3.3}

a. If $f$ is the function defined in (4), $\delta \in[0,1], \varepsilon>1,\left\{\lambda_{i}\right\}_{i \in N^{*}}$ is a sequence of positive numbers, such that $\lambda_{i+1}>\varepsilon \lambda_{i},(\forall) i \in N^{*}$ and $h$ is a measure function, satisfying the relation (5), then: $H_{h}(\Gamma)<+\infty$.

b. In the same hypothesis about $f$ and $\left\{\lambda_{i}\right\}_{i \in N^{*}}$, if $\delta>1$ and $\varepsilon>1$, then $H_{h}(\Gamma)<+\infty$.

Proof. The proof is analogous with that of the Theorem 2.2. (See [3].)

\section{References}

[1] Barbulescu, A., La finitude $d$ une h-mesure Hausdorff d'une ensemble de points dans le plan, Analele Universitatii "Valahia" Târgoviste, 1995/1996, fasc.II, 93-99.

[2] Barbulescu, A., P-module and p-capacity, Ph. D. Thesis, Iasi, 1997.

[3] Barbulescu, A., About the $h$ - measure of a set, Proceedings of the International Conference on Complex Analysis and Related Topics, Brasov, 2001 (to appear).

[4] Besicovitch, A.S., Ursell, H. D., Sets of fractional dimension (V): On dimensional numbers of some continuous curves, London Math. Soc.J., 12 (1937), 118-125. 